\title{
METODOLOGíA CAIT Y METODOLOGÍA SCHOLAS: PEDAGOGÍA DE LA INCLUSIÓN PARA EL ENCUENTRO
}

\author{
Miguel Ángel Barbero Barrios \\ Centro Universitario Sagrada Familia \\ Universidad de Jaén \\ mabarbero@fundacionsafa.es
}

Fecha de Recepción: 4 Enero 2019

Fecha de Admisión: 30 Abril 2019

\section{RESUMEN}

La metodología CAIT (Beltrán y Pérez, 2003) ha hecho una gran aportación a aquellos docentes que, desde la cotidianidad de su aula necesitaban un soporte teórico potente que pudiera vertebrar sus programaciones en base al proceso de aprendizaje humano de una forma bastante flexible y adaptable a distintas posibles condiciones contextuales y personales del alumnado. Por ello, una de las grandes aportaciones que realiza esta metodología es la de permitir que esas programaciones puedan convertirse en inclusivas al permitir que cualquier alumno pueda formar parte de las actividades propuestas. Podríamos decir que lo que en España y el mundo hispanohablante estamos llamando D.U.A. (Alba, 2012), adaptado del U.D.L. anglosajón -Universal Design for Learning(Kennette y Wilson, 2019) de alguna forma recoge lo que ya había sido formulado por otros autores y metodologías que intuyeron que el futuro de la innovación educativa vendría de la mano de programaciones inclusivas que potenciaran lo mejor de cada estudiante.

Por su parte, la metodología Scholas y la cultura del encuentro que está proponiendo como movimiento pedagógico a nivel internacional el Papa Católico Francisco (Fares, 2014) pretende justamente la participación de todos los alumnos, en todos los lugares y en todas las situaciones educativas posibles, promoviendo el encuentro con otros estudiantes diferentes (en cualquier ámbito) y su incidencia positiva y cooperativa en los diferentes contextos de los que provienen.

En esta comunicación se analizarán los puntos de conexión que existen en ambas metodologías y cómo el recorrido de la primera de las mencionadas puede ayudar a la segunda. En otras palabras: cómo las estrategias de aprendizaje de los procesos de sensibilización, elaboración y personalización que propone la CAIT pueden ayudar y encontrar correspondencia en el aprendizaje con corazón, cabeza y manos del que habla la propuesta metodológica internacional de Scholas.

Palabras clave: metodología CAIT; metodología Scholas; D.U.A.; aprendizaje inclusión 


\title{
METODOLOGÍA CAIT Y METODOLOGÍA SCHOLAS: PEDAGOGíA DE LA INCLUSIÓN PARA EL ENCUENTRO
}

\begin{abstract}
Methodology cait and methodology scholas: pedagogy of inclusion for the meeting. The CAIT methodology (Beltrán and Pérez, 2003) has made a great contribution to those teachers who, from their custom activity in the classroom needed a powerful theoretical support that could structure their programming based on the human learning process in a flexible and adaptable way, in order to better fix with what different students need. Therefore, one of the great contributions made by this methodology is to allow these programs to become inclusive by allowing any student to be part of the proposed activities. We could say that what we in Spain and the Spanish-speaking world are calling D.U.A. (Alba, 2012), adapted from the U.D.L. Anglo-Saxon -Universal Design for Learning(Kennette and Wilson, 2019) somehow collects what had already been formulated by other authors and methodologies that realized that the future of educational innovation would come from inclusive programming that would enhance the best of each student.

On the other hand, the Scholas methodology and the culture for encountering that the Catholic Pope Francisco (Fares, 2014) is proposing as an international pedagogical movement, is precisely the participation of all students, in all places and in all possible educational situations, promoting the encounter with other different students (in any field) and their positive and cooperative impact in the different contexts from which they come.

In this communication it will be analyzed the connection points between both methodologies and how the path of the first of these can help the second. In other words: how the learning strategies of the processes of sensitization, elaboration and personalization proposed by the CAIT methodology can help and find correspondence in the learning with heart, head and hands that the international methodological proposal of Scholas speaks about.
\end{abstract}

Keywords: CAIT methodology; scholas methodology; D.U.A.; learning, inclusion

\section{INTRODUCCIÓN}

Esta ponencia aborda el tema de la inclusión en educación desde la confluencia de dos metodologías que la fomentan; más aún, que abogan porque esta palabra sea innecesaria y sustituida simplemente por "educación". La una (CAIT) proviene del mundo académico y propone una sólida fundamentación teórica para la programación y evaluación de experiencias educativas y la otra (Scholas) proviene de experiencias concretas "de calle" (muchas de ellas, de calle, literalmente) que se están sistematizando y transfiriendo al mundo académico. En ese confluir, desde nuestro punto de vista, se produce un encuentro muy interesante que se convierte en sí mismo en objeto de análisis.

Las metodologías CAIT (Beltrán y Pérez, 2003) y Scholas (Bergoglio, 2013) articulan un modo de proceder que facilita la participación de todas las personas que forman parte de una comunidad educativa en torno a experiencias concretas, y lo hacen tanto desde el ámbito formal como no formal. Ahondar en las semejanzas y características propias de ambas propuestas resulta útil para la práctica educativa y esperanzador a partes iguales para aquellos que creemos en la inclusión, 0 mejor, en la educación.

La ponencia se divide en cuatro partes: una primera en la que se exponen ciertos consensos académicos al respecto del aprendizaje y se describe desde lo que se podría considerar como metodología inclusiva el modelo CAIT, una segunda en la que se describe la metodología Scholas como pedagogía del encuentro; una tercera en la que se analizan las confluencias entre CAIT y Scholas y una cuarta en la que se deja entrever cómo a partir de sus mutuos aportes se abre un futuro esperanzador para la inclusión, tanto desde el punto de vista de la investigación universitaria como desde la práctica educativa. 


\section{EL MODELO INCLUSIVO CAIT Y LA SECUENCIA DE APRENDIZAJE}

Existe en la comunidad científica consenso aceptado que define "aprendizaje" como un cambio más o menos permanente de conducta que se produce como resultado de la práctica (Kimble, 1971; Beltrán, 1993). La aceptación de la comunidad científica sobre esta definición viene dada por el carácter medible y cuantificable tanto de la conducta como de la práctica. Se acepta que puede haber un cambio observable en una conducta, en una práctica o ejercicio determinados y que ese cambio que lo provoca es duradero en el tiempo. Sin embargo, fuera de esos cambios observables, producto del aprendizaje, las diferencias entre unos enfoques y otros radica en la importancia otorgada a los procesos que llevan a ese resultado. Por un lado el conductista, que considera el aprendizaje como adquisición de respuestas y por otro el cognitivo, que considera el aprendizaje bien como adquisición de conocimiento o bien como construcción de significado. Se toman como referencia autores que explican el primer enfoque tanto desde un entendimiento clásico Skinner (1956) como contemporáneo Törneke (2016), de igual manera que el segundo (Goldberg, 1972). Estos distintos enfoques han ido dominando la esfera de la investigación en aprendizaje en los últimos años desde la mitad del siglo XX. En la actualidad, la visión del aprendizaje de la que parte la metodología CAIT que se expone bebe de la evolución del enfoque cognitivo, siempre enriquecido por las aportaciones de los demás enfoques, con sus correspondientes evoluciones, basadas en las nuevas aportaciones realizadas desde la psicología y la neurología y sus nexos de unión. Cuanto más claras son las investigaciones que vinculan ambas disciplinas, más difusos quedan los límites entre ellas.

El modelo CAIT es una herramienta de programación y evaluación útil para cualquier actividad educativa, que por serlo, lleva implícito un proceso de aprendizaje. Surge de la propuesta que realiza para toda la comunidad educativa el Foro Pedagógico de Internet de la Fundación Encuentro llamada CAIT (Constructiva, Autorregulada, Interactiva y Tecnológica), que a su vez se apoya en las investigaciones del Departamento de Psicología Evolutiva de la Facultad de Educación de la Universidad Complutense de Madrid dirigidas por los catedráticos Jesús Beltrán y Luz Pérez (2003). Esta metodología está basada en una serie de principios pedagógicos que se detallan a continuación (Martín, Beltrán y Pérez, 2003):

Parte de una "Pedagogía de la imaginación", que se centra en el alumno y que más que la reproducción de respuestas pretende la indagación o búsqueda.

Promueve un aprendizaje activo, constructivo, autorregulado y tecnológico frente a concepciones de carácter repetitivo.

Pretende aprender con la tecnología; es decir, entiende la tecnología como un instrumento cognitivo más que como herramienta de reproducción.

Transforma la información en conocimiento, dentro del contexto curricular correspondiente.

Pretende desarrollar el pensamiento analítico, el pensamiento pragmático y el pensamiento dialéctico a través del fomento de habilidades mentales.

Fomenta el desarrollo de los valores entendidos como los principios que regulan y establecen la convivencia pacífica entre los seres humanos.

Considera la creación de comunidades de aprendizaje como instrumentos de apoyo para el aprendizaje compartido y la negociación de significados.

Permite representar los contenidos del aprendizaje (resultados de los proyectos de trabajo realizados) mediante el uso de las habilidades verbales, orales y escritas.

Posee como ámbito de realización los niveles de infantil, primaria, secundaria y bachillerato.

Se trata de un modelo flexible, aunque se recomienda que se siga esta secuencia para la instrucción: objetivos, planificación, elaboración, personalización, aplicación y evaluación. 


\section{METODOLOGÍA CAIT Y METODOLOGÍA SCHOLAS: PEDAGOGÍA DE LA INCLUSIÓN PARA EL ENCUENTRO}

Estos principios apoyan una pedagogía a través de Internet que pretende ayudar a los alumnos a aprender de manera significativa. Lo que se espera de ellos es que sometan la información recogida de Internet a la acción del pensamiento, con el fin de analizar, relacionar, criticar, transferir y aplicar esa información, transformándola en conocimiento. Así, aprenden a aprender. Aprender no sólo significa adquirir información, sino desarrollar habilidades y destrezas que permitan seleccionar, organizar e interpretar la información. El aprendizaje al que nos lleva la pedagogía de Internet debe ser un aprendizaje activo, constructivo, contextualizado, interactivo y autorregulado. Todos estos principios favorecen sobremanera la inclusión de todos los alumnos de un grupo-clase en la actividad planteada, pues lo que se pretende no es la repetición, sino la investigación personal que cada cual (de forma individual y grupal) puede hacer.

El modelo CAIT describe siete parámetros como vertebradores del aprendizaje y guían las actividades del profesor y de los alumnos: contextualización, como escenario o contexto en el que se van a mover alumnos y profesores; planteamiento de objetivos; papel del profesor (más guía que contenedor de información); papel del alumno (protagonista del aprendizaje); instrumentos (se espera de los alumnos que sometan la información recogida de Internet (Alejaldre y Álvarez, 2018) 0 a través de otras herramientas pedagógicas a la acción del pensamiento, con el fin de analizar, relacionar, criticar, transferir y aplicar esa información, transformándola en conocimiento); desarrollo de actividades y procesos: consiste en planificar las tareas, seleccionar y organizar la información, actuar de forma crítica y creativa, transferir y aplicar los conocimientos; y evaluación (se evalúa la comprensión, la adquisición de estrategias, el aprender a aprender, la capacidad de autorregulación y las capacidades críticas e imaginativas).

El aprendizaje implica actividad en la que los alumnos se comprometen a realizar diferentes actividades para asimilar la información que reciben. Que el aprendizaje sea CONSTRUCTIVO significa que las actividades realizadas tienen como fin construir el conocimiento. Funcionará mejor si tiene lugar en contextos reales, por lo que tendrá que ser auténtico y situado. El conocimiento que se obtiene hoy en día sale de los libros y no se sabe muy bien cómo aplicarlo a la vida real si no es en Ios exámenes. Este exclusivo modo de proceder en nuestras escuelas frecuentemente desmotiva a los alumnos y disminuye las razones para aprender. Sin embargo, creemos que es posible introducir pequeños cambios en los que se contextualice en una situación real y se aprenda creando situaciones de partida motivantes para los estudiantes.

Debe ser AUTORREGULADO. Lo normal es que al principio, el aprendizaje sea controlado por el profesor, porque él sabe lo que hay que aprender y cómo hay que aprenderlo. Pero, a medida que el aprendizaje avanza, el profesor debe dejar al alumno el control sobre ese aprendizaje y actuar como mediador.

Y, por último, conviene que el aprendizaje sea INTERACTIVO. Esto permite a cada uno de los miembros del grupo construir el conocimiento de una manera propia y personal desde los puntos de vista que cada uno tiene sobre la información recogida. Todo esto se debe de poner en práctica dentro de un contexto TECNOLÓGICO, utilizando y canalizando de forma adecuada las tecnologías que han venido "para quedarse", que nos rodean queramos o no, y que junto a los tres rasgos anteriores definen el Modelo CAIT (Constructivo, Autorregulado, Interactivo y Tecnológico).

En definitiva, la metodología CAIT reúne todos estos principios y propone aplicarlos de forma abierta y flexible. Podríamos considerarlo, en este sentido, un modelo DUA -Diseño Universal de Aprendizaje- (Alba, 2012; Díez y Sánchez Fuentes, 2015) que permite que los diferentes alumnos de cualquier grupo que aprende encuentren expresión a sus distintos talentos y capacidades y muestren lo mejor de sí. 


\section{EL MODELO INCLUSIVO SCHOLAS Y LA PEDAGOGÍA DEL ENCUENTRO}

La metodología Scholas y la cultura del encuentro que está proponiendo como movimiento pedagógico a nivel internacional el Papa Católico Francisco (Fares, 2014) pretende justamente la participación de todos los alumnos, en todos los lugares y en todas las situaciones educativas posibles, promoviendo el encuentro con otros estudiantes diferentes (en cualquier ámbito) y su incidencia positiva y cooperativa en los diferentes contextos de los que provienen.

Lo que podríamos llamar pedagogía "Scholas" encuentra sus raíces en el pensamiento del Papa Francisco. Es fácil encontrar en sus escritos multitud de referencias al mundo de lo educativo, hasta el punto de ser considerado como el Papa de la educación (Domingo, 2019). Pero este legado no estará solo compuesto por puro pensamiento. Hay acción. De hecho, es una de sus implicaciones claras. En las propias reflexiones de Francisco va implícito su carácter práctico. Es decir, podríamos decir que piensa que el mejor camino de la pedagogía se demuestra "andando" (Fares, op.Cit.). Scholas, incluso antes de tener nombre ya fue una experiencia que encontró su origen en la ciudad de Buenos Aires en 2001, en tiempos en los que Jorge Mario Bergoglio era Arzobispo. Trató de asumir el reto de crear conexión entre las realidades de jóvenes de distintos barrios que tenían poco que ver entre sí y entre los que estaba aumentando la problemática social en forma de prejuicios, violencia, adicción a sustancias nocivas y alcoholismo entre otros. Se apoyó en la tecnología para construir conexión y conocimiento mutuo entre ellos, creando experiencias de conocimiento y hermandad entre centros educativos. La empatía entre grupos sociales muy distantes creció; fueron construyendo puentes. Gracias a la implicación en este proceso de los distintos agentes educativos bajo el paraguas de una iniciativa que se denominó "Escuelas Hermanas" desde variados frentes (familia, mundo académico escolar y universitario, ámbito no formal, administración local e iglesia y distintas confesiones religiosas) todos empezaron a remar en la misma dirección para cambiar pequeñas cosas del entorno de los barrios más necesitados. El éxito fue tal que a través de esta herramienta social los jóvenes de esos barrios llegaron a realizar varias iniciativas que se aprobaron en el congreso argentino.

Cuando Jorge Mario Bergoglio fue elegido Papa, los colaboradores de aquella iniciativa se preguntaban qué iba a ser de la iniciativa "Escuelas Hermanas" cuando el arzobispo de Buenos Aires ya no estuviera allí. Pero, más allá de desaparecer, amplió su horizonte a todo el mundo convirtiéndose en lo que después fue Scholas Occurrentes. Un proyecto mucho más amplio pero que conservaba la esencia de esa experiencia inicial: que desde la misma calle 0 aula las iniciativas de los jóvenes pudieran llegar a los estamentos más amplios y cambiar cosas que parecerían imposibles si no se actuara en red. En otras palabras, que el "empoderamiento" de los jóvenes pudiera encontrar un apoyo aún mayor en una iniciativa más global. En 2015 Scholas se constituyó en Fundación Pontificia.

Las iniciativas de Scholas siempre han promovido encuentros entre personas de características, labores y contextos muy diferentes. Es por esto que puede hablarse de que aboga de una forma decidida por una pedagogía del encuentro. El encuentro de lo alejado puede ser un trabajo de gran dificultad, de ahí que este sea precisamente el mayor reto educativo. El pacto educativo, en cualquier nivel educativo, desde esta perspectiva, se convierte en el horizonte clave para este proyecto.

Teniendo como centro la dignidad y el desarrollo de la persona (Bergoglio, op. Cit.), la pedagogía Scholas propone el aprendizaje de los niños y jóvenes desde tres pilares fundamentales que no tienen por qué estar alineados exactamente con los currículums oficiales de los sistemas educativos (por no tener que depender de ellos) si bien sí que se proponen como ejes a explorar desde todos los ámbitos y contextos educativos: se trata del deporte, el arte y la tecnología. Estos son los principales campos de expresión de los jóvenes y niños y por ello, conforman los pilares básicos 


\section{METODOLOGÍA CAIT Y METODOLOGÍA SCHOLAS: PEDAGOGíA DE LA INCLUSIÓN PARA EL ENCUENTRO}

para los contenidos y habilidades a explorar desde Scholas. A su vez, para trabajar a partir de ellos se propone una metodología que dentro de cada eje mencionado pase por:

La motivación inicial hacia el aprendizaje enfocado hacia una realidad contextualizada susceptible de ser mejorada (poniendo en marcha los afectos), lo que el Papa llama, trayendo al escenario actual las palabras de San Francisco, "el corazón".

La exploración cognitiva sobre el problema, de los propios conocimientos y de cómo estos pueden contribuir a sus posibles soluciones ("la cabeza").

La acción para poner en marcha los talentos que puedan traer mejoras a los seres humanos con quien se comparte la vida (en lenguaje de Francisco, "las manos").

Una concreción de lo que podríamos llamar modo de proceder "Scholas" lo encontramos de modo paradigmático en la experiencia "Scholas Ciudadanía", que pone en conocimiento a alumnado de edades comprendidas entre los 14 y 16 años procedente de centros educativos de muy distinta índole para que trabajen sobre los temas que más les importan y les propongan soluciones. La tríada corazón, cabeza y manos se trabaja con los alumnos a través de una completa propuesta de actividades que durante seis días articula de forma armónica otra triada: pensamiento, arte y juego, donde los propios alumnos pueden elegir dónde se sienten más cómodos y desde dónde pueden aportar más.

Esta experiencia es también paradigmática para visualizar la inclusión en Scholas, pues nadie queda fuera de las actividades. Siempre se invita a expresar, posibilitando que se haga desde cualquier medio o instrumento y desde cualquier capacidad, por lo que no se cierra la puerta a las personas que en otros ámbitos pudieran pasar por disruptivas, indisciplinadas o molestas, por lo que podríamos considerar la pedagogía de Scholas como típicamente inclusiva (Echeita, 2017).

La pedagogía Scholas no es solo una herramienta educativa al servicio del alumnado o el aprendizaje, ni siquiera al servicio de la acción social. Intenta ir más allá. Intenta construir puentes entre las personas para generar buena convivencia allí donde es difícil, desde la consciencia de que, precisamente en la educación, los sistemas educativos actuales encuentran enormes franjas de ruptura. En España, de hecho, tenemos un caso paradigmático: estamos ante un sistema educativo cambiante, al arbitrio de los gobernantes de turno, ideologizado, instrumento de réditos políticos y con un futuro de unidad que muchos dan ya por imposible (Domingo, op. Cit.). No obstante, lejos de ese pensamiento de desánimo, que a pesar de que pueda albergar sus razones, desde Scholas se pretende retomar el pacto educativo y que sean los propios agentes que intervienen en la planificación de la educación (administración de distintos niveles, desde lo estatal hasta lo local, pasando por el profesorado, las familias y el alumnado) sean capaces de llegar a un consenso por el cual se articule de una vez por todas un sistema educativo estable que sobre todo, busque el beneficio y la cohesión social, desde el respeto a las diferencias que inevitablemente surgirán en el proceso, propias de las mismas diferencias de los actores participantes en el mismo. En este contexto, nos parece especialmente oportuno que existan iniciativas que caminen hacia ello y que no se den por vencidas porque hay mucho en juego.

\section{DE LA UNIVERSIDAD A LA PRÁCTICA (CAIT) Y DE LA PRÁCTICA A LA UNIVERSIDAD (SCHOLAS). DOS REALIDADES NECESARIAS Y COMPLEMENTARIAS PARA EL PRESENTE Y FUTURO}

La metodología CAIT es el producto de un proceso de varios años de investigación universitaria que en torno a constructos tan importantes para el mundo educativo como la inteligencia, las estrategias de aprendizaje, la instrucción inclusiva o la secuenciación de los procesos de aprendizaje (Barbero y Beltrán, 2014) generó un modelo teórico concreto articulado (y por ello aplicable) de intervención educativa inclusiva. Scholas, por su parte, surge de la iniciativa "a pie de calle" de per- 
sonas preocupadas por mejorar la situación de los niños y jóvenes pertenecientes a entornos deprimidos que no encontraban en el sistema educativo actual un modo de expresión de su talento que, desde la práctica educativa fueron encontrando mecanismos de acción social cada vez más relevantes y en red.

En perspectiva, podríamos apuntar a que el recorrido de la CAIT, que en sí mismo como modelo sigue avanzando desde las nuevas teorías e instituciones que se ocupan de su desarrollo ante todo lo que puede seguir aportando (Barbero, 2017), supone un excelente marco de acción para la pedagogía Scholas. En otras palabras, que los maestros de la práctica educativa que están uniendo a escuelas de todo el mundo a través de los programas de Scholas pueden encontrar en la CAIT una elaborada herramienta para el trabajo en cada uno de sus pilares u objetivos marcados. El triángulo de la actividad humana de Morton y Weinstein (2002) nos aporta pista definitiva que explica la unidad de las dos triadas básicas de cada modelo bajo lo que ellos llaman "instancias de la actividad humana", a saber: querer, poder y decidir:

Figura 1: Instancias fundamentales para la realización de cualquier actividad (Morton y Weinstein, 2002)

$$
\text { Decidir (toma de decisiones) }
$$

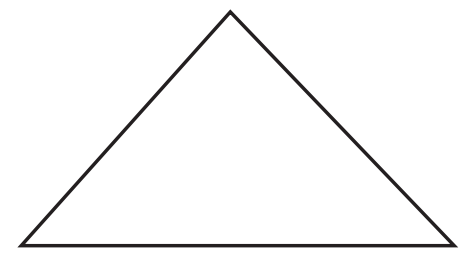

Querer (voluntad)

Poder (capacidad)

Estas dimensiones encuentran su correspondencia el el modelo de instrucción CAIT que intenta articular su lógica en la práctica instruccional de un educador, enfocando el "querer" en procesos de motivación; el "poder" en procesos de elaboración y el "decidir" en procesos de "personalización", los tres autorregulados por el alumno a través de procesos de metacognición (Beltrán y Vega, 2003). Para cada uno de estos niveles, a su vez, los pilares del modelo Scholas encuentran su acomodo, pues aportan a ese modelo de instrucción inclusivo la lógica pedagógica del encuentro tal y como se puede resumir en el siguiente esquema básico de acción conjunta CAIT- Scholas:

Educamos desde el CORAZÓN: A través del fomento de estrategias de sensibilización (aumentar la motivación en niños o jóvenes apasionados por el deporte, el arte o la tecnología).

Educamos desde la CABEZA: A través del fomento de estrategias de elaboración que proyecten el rendimiento académico 0 el aprendizaje no formal desde distintos estilos de pensamiento.

Educamos desde las MANOS: A través del fomento de estrategias que permitan la elaboración personalizada de productos finales DUA que puedan compartirse y que generen un beneficio a la comunidad de Scholas Occurrentes 0 de otras redes o contextos.

\section{CONCLUSIÓN Y PROSPECTIVA}

Encontramos que la cultura del encuentro es en sí misma una propuesta privilegiada de psicología positiva. Es por ello que la participación en el Simposio del Congreso INFAD en Salamanca 2019 supone para el equipo de la Cátedra Scholas Ocurrentes una oportunidad para dar a conocer 


\section{METODOLOGÍA CAIT Y METOdOLOGÍA SCHOLAS: PEDAGOGÍA DE LA INCLUSIÓN PARA EL ENCUENTRO}

en el mundo académico una experiencia eminentemente práctica, la de Scholas, que está moviendo los cimientos de algo grande: la conexión de escuelas de todo el mundo; al mismo tiempo, también nos ha permitido dar a conocer el enfoque y la fundamentación teórica de futuros estudios a los que la conexión, o mejor, el puente "mundo académico-Scholas" aconseja transitar. Estos pondrán en valor el gran trabajo realizado por los teóricos de la CAIT y las luces que para la inclusión y los modelos DUA aportaron Jesús Beltrán, Luz Pérez y José María Martín Patino (presidente y fundador precisamente de la Fundación Encuentro), la propia asociación INFAD y sus colaboradores durante tantos años. Gracias por la atención prestada a estas palabras y a su sentido. Seguiremos en el empeño.

\section{REFERENCIAS BIBLIOGRÁFICAS}

Alba Pastor, C. (2012). Aportaciones del Diseño Universal para el Aprendizaje y de losvmateriales digitales en el logro de una enseñanza accesible, en Navarro, J., Fernández, Ma T., Soto, F. J. y Tortosa F. (coords.) (2012). Respuestas flexibles en contextos educativos diversos. Murcia: Consejería de Educación, Formación y Empleo.

Alejaldre, L. y Álvarez, E. (2018). Estrategias metacognitivas para la lectura en línea: estudio de caso de estudiantes tailandeses de ELE universitarios. Aula de Encuentro, 2, 20, pp. 140-170. D0I: https://dx.doi.org/10.17561/ae.v20i2.8

Barbero, M.A. (2017). Estrategias de aprendizaje y rendimiento a través de la metodología CAIT (Tesis Doctoral). Universidad Complutense de Madrid: Madrid.

Barbero, M.A. y Beltrán, J.A. (2014). El modelo inclusivo CAIT. Un modelo de intervención en estrategias de aprendizaje y rendimiento a través de la metodología CAIT. INFAD - VII Congreso de Psicología y Educación. Badajoz, 9-12 de abril. 2014, 1 VOL.7, 589. Recuperado de: http://infad.eu/RevistalNFAD/index.php/publicaciones/revista-infad-2014/no1-volumen-7-pp.$\underline{589 /}$

Beltrán, J.A. (1993). Procesos, estrategias y técnicas de aprendizaje. Madrid: Síntesis.

Beltrán, J.A. y Pérez, L. F. (2003). De la Pedagogía de la memoria a la Pedagogía de la imaginación. La novedad pedagógica de Internet. Madrid: Fundación Encuentro.

Beltrán, J.A. y Vega, M. (2003). Aprender con tecnología en el Aula Inteligente. En F. Segovia: El Aula Inteligente: Nuevas perspectivas. Madrid: Espasa Calpe.

Bergoglio, J.M. (2013). Educar: exigencia y pasión.Desafíos para educadores cristianos. Buenos Aires: Publicaciones Claretianas.

Díez, E. y Sánchez Fuentes, S. (2015). Diseño universal para el aprendizaje como metodología docente para atender a la diversidad en la universidad. Aula Abierta, Vol. 43 Issue 2 JulyDecember 2015, pp. 87-93. DOI: https://doi.org/10.1016/j.aula.2014.12.002

Domingo, A. (2019, 10 abril). Bases antropológicas del pacto educativo. Despertar el compromiso educativo para un humanismo solidario. En Cañizares (Presidente). I/ Congreso Internacional Scholas Occurrentes. Universidad Católica de Valencia, Valencia.

Echeita, G. (2017). Educación inclusiva. Sonrisas y lágrimas. Aula Abierta, 46, 17-24. Recuperado de, https://www.unioviedo.es/reunido/index.php/AA/issue/view/917

Fares, D. (2014). Papa Francisco. La cultura del encuentro. Barcelona: Edhasa.

Goldberg, L. R. (1972). Student personality characteristics and optimal college learning conditions: An extensive search for trait-by-treatment interaction effects. Instructional Science, 1 (2), 153210.

Kimble, G. A. (1971). Cognitive inhibition in classical conditioning. Essays in neobehoviorism, 69

Martín, J. M., Beltrán, J.A., y Pérez, L. (2003): Cómo aprender con Internet. Madrid: Foro Pedagógico de Internet. 
PSICOLOGÍA POSITIVA

Morton, L. y Weinstein, J. (2002). Stuck in a Rut: The Role of Creative Thinking in Problem Solving and Legal Education. Clinical L. Rev., 9, 835.

Skinner, B.F. (1957). Verbal behavior. Nueva York: Appleton Century Crofts.

Törneke, N. (2016). Aprendiendo TMR. Úbeda: Didacbook. 
